\title{
The Altered Water System: Excess Levels of Free Radicals Contribute to Carcinogenesis by Altering Arginine Vasopressin Production and Secretion and Promoting Dysregulated Water Homeostasis in Concert with Other Factors
}

\author{
Amy Marie Beutler and Bradford N. Strand \\ North Dakota State University (NDSU), c/o, Department 2620, P.O. Box 6050, Fargo, ND 58102-6050, USA \\ Correspondence should be addressed to Amy Marie Beutler; amy.m.beutler@ndsu.edu \\ Received 13 September 2014; Revised 13 November 2014; Accepted 14 November 2014; Published 26 November 2014 \\ Academic Editor: Chih-Hsin Tang \\ Copyright (c) 2014 A. M. Beutler and B. N. Strand. This is an open access article distributed under the Creative Commons \\ Attribution License, which permits unrestricted use, distribution, and reproduction in any medium, provided the original work is \\ properly cited.
}

\begin{abstract}
A large body of evidence accumulated during the last decade has revealed diverse roles of dysregulated water homeostasis in tumorigenesis. In particular, many tumors hypersecrete arginine vasopressin (AVP) causing hypoosmolar conditions associated with different cancers. Excess levels of free radicals and nonosmotic stimuli may act as signals in water homeostasis and induce the production and secretion of AVP. Hypoosmolar conditions cause alterations in the expression of many genes. Other alterations in hydration patterns may induce mutations and increase the levels of protein kinases to contribute to oncogenesis. Furthermore, AVP regulates the hypothalamic-pituitary-adrenal axis and angiogenesis, and its overproduction may contribute to tumor growth and metabolism. This review article describes a mechanism by which oxygen radical species and other free radicals act as signaling molecules that, in concert with increased AVP production and secretion, pleiotropically affect tumor growth and metabolism, resulting in dysregulated proliferation, cell cycle arrest, apoptosis, and genomic instability.
\end{abstract}

\section{Introduction}

Previously unrecognized vital roles of water in the body have been uncovered during the last decade $[1,2]$. In the case of humans, water molecules are hypothesized to behave as a "molecular supercomputer" that interprets and reacts to biochemical pathways in accordance with prevailing demands to achieve homeostasis through complex signaling pathways that are referred to as the "water governing cycle" [2].

Plants possess complex signaling pathways that are highly adaptable because of their ability to interpret and react to stressors such as osmotic fluctuations and the overabundance of saline by rapidly altering gene expression that corresponds to biochemical and physiological alterations, even under mild conditions, to achieve homeostasis [3-5]. However, limited research has addressed whether osmotic fluctuations rapidly alter gene expression in accordance with prevailing demands in animals and humans. For example, research on animals has shown that sustained hypoosmolar (excess water relative to solute molecules) or hyperosmolar conditions (water deficiency relative to solute molecules) significantly alter the expression of a wide variety of regulatory genes in a global but selective manner $[6,7]$.

Moreover, water molecules contribute to specific and nonspecific binding of peptides to proteins $[1,8]$, may play a significant role in mediating sequence-specific recognition, and increase the affinity of proteins for DNA [9-15]. The role of water in protein-DNA interactions is multifaceted [11]; however, a complete summary is beyond the scope of this review. Generally, protein-DNA complexes are very diverse in their use of water [11]. Three general classes of water molecules have been recognized: (a) molecules that do not interact, (b) molecules that interact with the surface of macromolecules, and (c) molecules that interact in deep 
crevices or interior cavities [11]. Surface water molecules are somewhat well ordered and may exist in partially ordered hydration patterns. Interior water molecules are significantly ordered by means of extensive interaction with dissolved macromolecules [11]. A large body of literature concerning the hydration patterns of proteins and nucleic acids indicates that surface hydration of proteins is precise with respect to local stereochemistry. Furthermore, the local hydration patterns of DNA vary according to the DNA sequence [11]. All proteins displace water molecules upon binding to DNA, which creates an entropic change in free energy. Some water molecules remain at the interface to mediate a specific reconnection that facilitates the formation of a dynamic network of hydrogen bonds between proteins and DNA [11]. For nonspecific recognition, the extra water molecules may allow proteins to search along DNA for a binding site [11]. Furthermore, water molecules, with the help of a hydrogenbond frame shift, can fill the gap at the N6 atom of adenine when combined with guanine, thereby mimicking ATP. The use of water molecules to create a backbone structure when combined with GTP mimics ATP, enabling dual coenzyme specificity of protein kinase production $[16,17]$. Increased levels of the protein kinase CK2 are found in many cancers [16].

Dysregulation of water homeostasis may be associated with oncogenesis. For example, dysregulated water homeostasis alters gene expression by rapidly upregulating genes such as LMO-4 that are associated with cancer $[6,7]$, alters hydration patterns to cause promiscuous binding of peptides to proteins and mutations $[1,8]$, and increases protein kinase production to contribute to oncogenesis and tumor progression [16]. Additionally, hypoosmolality is associated with many different cancers, including pulmonary, pancreatic, renal, nasopharyngeal, duodenal, urethral, prostatic, uterine, and gastric cancers, as well as leukemia, mesothelioma, thymoma, and Hodgkin lymphoma [18]. In these cancers, tumors hypersecrete arginine vasopressin (AVP), causing hypoosmolarity [18]. However, it has not been determined whether hypoosmolar conditions produce tumors prior to tumor secretion of AVP.

AVP, which is the primary controller of water homeostasis [18], is stored in the posterior pituitary gland, and changes in plasma osmolarity primarily control AVP production and secretion; however, AVP levels can also be regulated by blood pressure, which is not typically associated with cancer risk $[19,20]$. However, molecules and conditions that do not alter osmolarity but still stimulate AVP production and secretion include but are not limited to $[19,20]$ reactive oxygen species (ROS) $[21,22]$, tobacco smoke and nicotine [23-25], stress [26], and excessive alcohol consumption [27]. These nonosmotic factors are considered to serve as signals that regulate water homeostasis $[21,22]$ and are commonly associated with an increased risk for cancer. Smoking and administration of nicotine have been shown to release AVP proportionally to the amount of nicotine that enters the blood $[23,25]$, especially in elderly populations, possibly suggesting a disorder of AVP production in the elderly [24].

The purpose of this review is threefold. First, it compiles a growing body of experimental evidence showing the many roles that dysregulated water homeostasis plays in tumorigenesis. Second, although hypoosmolar conditions can be caused by other conditions such as reduced mineralocorticoid levels, this paper examines how the hypersecretion of AVP causes hypoosmolarity and dysregulation of other processes that may contribute to the growth and metabolism of tumors. Third, following this model, a mechanism is presented by which one of many signaling molecules associated with AVP, specifically ROS, synergizes with AVP production and secretion to induce pleiotropic effects that maintain a dysregulated state, thereby contributing to tumor pathogenesis.

\section{Alterations in Water Balance Lead to Alterations of Protein and DNA Expression and Function, Dual Coenzyme Specificity, and Changes in Gene Expression}

The role of water in influencing chemical reactions, metabolism, protein synthesis and function, DNA synthesis, and transcription leads to the conclusion that water is the "unsung" universal regulator of the cell [2] and that studying the diffusion of water throughout the body may improve our understanding of how the body responds to disease [28]. For example, patients with cancer frequently have excess body water (hypoosmolarity) that is attributable to the hypersecretion of AVP and dysregulates $\mathrm{pH}$ and electrolyte homeostasis [29]. Hypoosmolarity alters the balance of electrolytes and $\mathrm{pH}$ and the presence of extra water molecules where proteins and DNA interface can lead to harmful mutations [11], indicating that water homeostasis is essential for maintaining genomic stability.

Water molecules facilitate specific and nonspecific binding of peptides to proteins $[1,8]$. Nucleic acids and proteins have specific, complementary hydration patterns that suggest their importance for DNA and protein recognition. Furthermore, water plays a significant role in mediating sequencespecific recognition and increases the affinity of proteins for DNA [9-15]. The interaction of water molecules with certain proteins, such as OppA, greatly favors nonspecific binding, which indicates that the ability of water to neutralize electrostatic charge and to alter hydrogen-bonding patterns facilitates the binding of peptides that contain commonly occurring amino acids to proteins [8]. Rather than inducing "promiscuous binding," water contributes to the highly selective binding of ligands to certain proteins, revealing the diverse effect of water molecules on protein-DNA binding [8].

Water molecules contribute to dual coenzyme specificity that increases the activation of protein kinases [17]. For example, water molecules create a backbone structure that enables GTP to mimic the conformation of ATP that binds to the casein kinase 2 (CK2) activation site [17]. The use of ATP and GTP as energy sources for increasing protein kinase activity significantly increases cellular growth, proliferation, and the potential for malignant transformation [16]. Moreover, the regulation of water influences gene expression by animal and plant cells. Sustained hypoosmolar conditions alter the expression of certain genes, including genes that contribute to 
cancer [6]. For example, rats subjected to sustained hypoosmolar conditions showed significantly altered expression of 1,564 genes, of which 657 were downregulated and 907 were upregulated [6]. In particular, expression of LMO-4 radically increased by more than 2-fold under hypoosmolar and hyperosmolar conditions. Moreover, LMO-4 is significantly overexpressed in over $50 \%$ of all primary breast cancers and may cause sporadic breast cancer by downregulating the activity of the tumor suppressor BRCA1 [30]. Such changes in gene expression may represent specific osmotic regulation [6]. Interestingly, all breast cancers express $A V P$, in contrast to normal breast tissue [31, 32].

Hypoosmolar conditions increase the expression of estrogen receptor B mRNA and glucocorticoid receptors [7]. Increased expression of the receptors for estrogen and glucocorticoids significantly increases the likelihood of developing cancers such as those of the breast and uterus [33]. For example, the expression of estrogen receptor B mRNA increases in rats chronically exposed to hypoosmolality, whereas chronic hyperosmolality decreases the expression of estrogen receptor B mRNA [7]; however, increased expression of estrogen receptor B mRNA under conditions of hypoosmolarity has not been verified [6]. Further research is required to determine whether increased estrogen receptor levels are a specific consequence of osmolarity.

\section{AVP and Its Diverse Effects on Carcinogenesis}

AVP is a peptide hormone that is mainly produced in the hypothalamus and acts peripherally and centrally throughout the body, causing antidiuresis, vasoconstriction, hemostasis, corticotrophin release, and hepatic glycogenolysis [19]. Moreover, AVP interacts with hormones and the components of certain regulatory pathways, and its central effects may influence behavior [19]. The three AVP receptors V1a-R, V1b$\mathrm{R}$, and $\mathrm{V} 2-\mathrm{R}$ are expressed on the surface of pulmonary, pancreatic, and breast cancer cells [34]. An additional study found that small cell lung cancer and breast cancer express the vasopressin gene and an abnormal form of the V2 receptor in addition to the three normal receptors [35]. Normal V2-R is primarily responsible for regulating water, and AVP acts as an antidiuretic through V2-R to induce the synthesis of aquaporin 2 (AQP2) [19, 20]. Classical small cell lung cancer and breast cancer were found to express both normal and abnormal forms of V2-R [35]. The abnormal form of V2receptor present in cancer cells was found to contain the normal sequence for V2-R with a complete additional 106 bases of intron 2 and is thus thought to be a C-terminally truncated protein [35]. Furthermore, overproduction of AVP and its receptors has diverse effects on tumor growth and metabolism, and AVP may act as an autocrine growth factor in breast and lung cancers $[31,32]$. The production of AVP by tumors may be required for oncogenic transformation [33]. Vasopressin has been found to inhibit the growth of breast cancer cells, possibly through effects on V2-R, which is supported by the observed interaction of oxytocin (an antagonist of vasopressin) with the V2-R [35].
AVP is stored in the posterior pituitary gland. Changes in plasma osmolarity are sensed by osmolar receptors, which may be present outside the blood-brain barrier, to control AVP production and secretion [19, 20]. However, nonosmotic events and molecules $[19,20]$ such as ROS $[21,22]$, tobacco smoke and nicotine [23-25], stress [26], and excessive alcohol consumption [27] also stimulate AVP production and secretion. More recent evidence suggests that ROS increase AVP production and secretion [21, 22] by the hypothalamoneurohypophyseal complex and through the noradrenaline-NO pathway, indicating that ROS and other free radicals act as signaling molecules that regulate various physiological processes of AVP, such as water homeostasis. Therefore, free radicals appear to be more than just cytotoxic molecules [22].

AVP acts as the primary controller of water homeostasis by regulating water retention and diuresis [20]. Binding of AVP to V2 receptors induces production of the membrane protein aquaporin 2 (AQP2) [34], which is expressed by cells of the renal collecting ducts and most other cell types [36]. When AVP binds to AQP2, water is transported through large channels in cell membranes that were previously unavailable and causes the absorption of abnormally high volumes of water [35]. The hypersecretion of AVP dysregulates water homeostasis by inducing hypoosmolality [20]. Elevated AVP levels cause antidiuresis, and decreased AVP levels cause diuresis [20]. However, other factors may cause hypoosmolality, such as elevated plasma atrial natriuretic peptide (ANP) levels, which are associated with inappropriately low AVP levels that increase solute depletion, although this phenomenon is not common [18] and is not the focus of this review. AVP also aids in the regulation of the adrenalstress axis through the V1b receptor [37], which involves the synthesis and release of glucocorticoids from the adrenal cortex [19].

Glucocorticoids are required for maintaining homeostasis [38]. Chronic high levels of glucocorticoids exert detrimental effects such as inhibiting certain immune functions involving cytokines and natural killer cells, which protects tumors from the immune system [39]. Diminished immunosurveillance plays a critical role in allowing cancers to grow and spread [40]. Additional research is required to verify that hypersecretion of AVP detrimentally affects the immune system through chronic high glucocorticoid production. The specific role of AVP in the HPA axis is controversial because of the use of indirect or correlationbased studies [41]. AVP appears to play diverse roles in the HPA axis ranging from immunoneutralization inhibiting the increase in plasma ACTH to advantageous release rather than corticotropin-releasing hormone $(\mathrm{CRH})$ in response to acute and chronic stressors [41]. In these studies, chronic stress resulted in downregulation of $\mathrm{CRH}$ and increased the sustained transcription of $A V P$, possibly to compensate for the decreased level of CRH $[26,41]$.

Furthermore, under stress, the body utilizes glucocorticoid production as a form of energy that aids in angiogenesis [42]. Thus, hypersecretion of AVP causes an increase in glucocorticoid levels, which also stimulate angiogenesis as a secondary effect. Moreover, AVP directly causes local 
tissue hypoxia in rats that increases angiogenesis, strongly suggesting that AVP may also participate in angiogenesis by causing the restriction of afferent arterioles and the activation of perivascular V1a receptors [43]. Future research is required to determine whether AVP functions similarly in humans.

\section{Oxidative Stress May Dysregulate AVP Production and Secretion}

When ROS are present at nonphysiological concentrations, they are extremely toxic and may be lethal $[44,45]$. The human body possesses an antioxidant defense system that regulates the levels of ROS to prevent toxicity. However, this balance is easily tilted in favor of oxidative stress [4648] under the following conditions: (1) inadequate levels of antioxidants derived from the diet and (2) excessive production of ROS induced by factors such as exposure to toxins, drugs that increase the levels of ROS, excessive free radical production, and diseases that induce secondary oxidative stress [46-48].

Because high levels of ROS are readily generated and ROS may act as a signal for AVP production and secretion, a question arises regarding whether high levels of ROS dysregulate expression of AVP, which synergizes with ROS, thereby causing the dysregulation of water homeostasis and other functions that lead to carcinogenesis. The same question arises regarding tobacco smoke, stress, excessive alcohol consumption, and other nonosmotic conditions that stimulate AVP production and secretion. Thus, it is important to investigate whether dysregulation of water homeostasis caused by an imbalance in the levels of signaling molecules alters the "water governing cycle" and contributes to carcinogenesis.

\section{Excessive ROS and AVP Synergize to Contribute to Oncogenesis}

High levels of ROS cause cancer through different mechanisms such as damage to DNA, proteins, and lipids [45, 49]. For example, ROS inactivate TP53, which inhibits apoptosis [45]. High ROS levels also increase histone acetylation, which facilitates oncogenesis by damaging chromatin [50, 51]. Moreover, ROS directly increase the production of thioredoxin $[52,53]$, which increases the growth of many tumor cell lines and prevents cell death in the presence of chemotherapeutic drugs by inhibiting signaling mechanisms that induce apoptosis $[53,54]$.

Excess levels of ROS directly affect DNA and other molecules involved in oncogenesis, and ROS act as signaling molecules that induce the hypersecretion of AVP, which may directly or indirectly contribute to oncogenesis. For example, overproduction of AVP can lead to hypoosmolar conditions [20]. Hypoosmolar conditions contribute to oncogenesis by altering the expression of certain genes, including the LMO4 oncogene $[6,7]$, thereby altering the hydration patterns required for DNA and protein recognition $[1,8,11]$ and enabling dual coenzyme specificity that increases protein kinase production to levels that promote oncogenesis $[16,17]$.
In concert with the V1b receptor, hypersecretion of AVP causes production of glucocorticoids, favoring the growth and spread of cancer cells by supporting angiogenesis, although further research is required to verify the effect on immune function.

Furthermore, overproduction of AVP and its receptors influences tumor growth and metabolism, and AVP may act as an autocrine growth factor in some cancers [31, 32, 34]. The following list summarizes how the synergy of high levels of ROS and AVP production and secretion maintains a balance among dysregulated proliferation, cell cycle arrest, apoptosis, and genomic instability. A possible explanation for why cancers caused by ROS, which increase AVP production and secretion to abnormal levels, maintain a dysregulated state is as follows: many of the factors that give rise to symptoms of pregnancy, such as increased AVP, high levels of thioredoxin, decreased immune function, increased angiogenesis, increased glucocorticoid levels, and increased blood volume, are influenced by dysregulated ROS and AVP. Figure 1 illustrates a possible mechanism by which excessive ROS and AVP synergize, thereby creating conditions that contribute to oncogenesis and may lead to metastasis.

Synergism between Dysregulated ROS and AVP Evades Cellular Control Mechanisms to Contribute to Oncogenesis

Dysregulated proliferation: hypoosmolar conditions significantly alter the expression of over 1,500 genes, including more than 2-fold upregulation of the LMO4 oncogene. Hypoosmolar conditions may upregulate genes involved in the transcription of the genes encoding the receptors for estrogen and glucocorticoid receptors, which increases the risk of breast and uterine cancers.

Cell growth: excess water molecules increase protein kinase production, which rapidly induces an increase in cell growth. Moreover, oxidative stress induces the synthesis of high levels of thioredoxin, which stimulates the growth of many tumor cell lines.

Inhibition of apoptosis: high levels of oxidative stress lead to damage to TP53 and increase the inhibition of apoptotic signaling pathways by thioredoxin.

Genomic instability: changes in hydration patterns may alter DNA, protein, and peptide binding recognition, leading to deleterious mutations. Moreover, high levels of ROS damage DNA, proteins, and lipids and increase the acetylation of histones, which may damage chromatin.

Survival: AVP activates glucocorticoid production that contributes to angiogenesis, favoring the growth and spread of cancer. AVP may stimulate angiogenesis by restricting afferent arterioles and activating perivascular V1a-R. Further research is needed to verify the role of AVP in inhibiting immune functions via sustained glucocorticoid production, thereby facilitating cancer survival. 


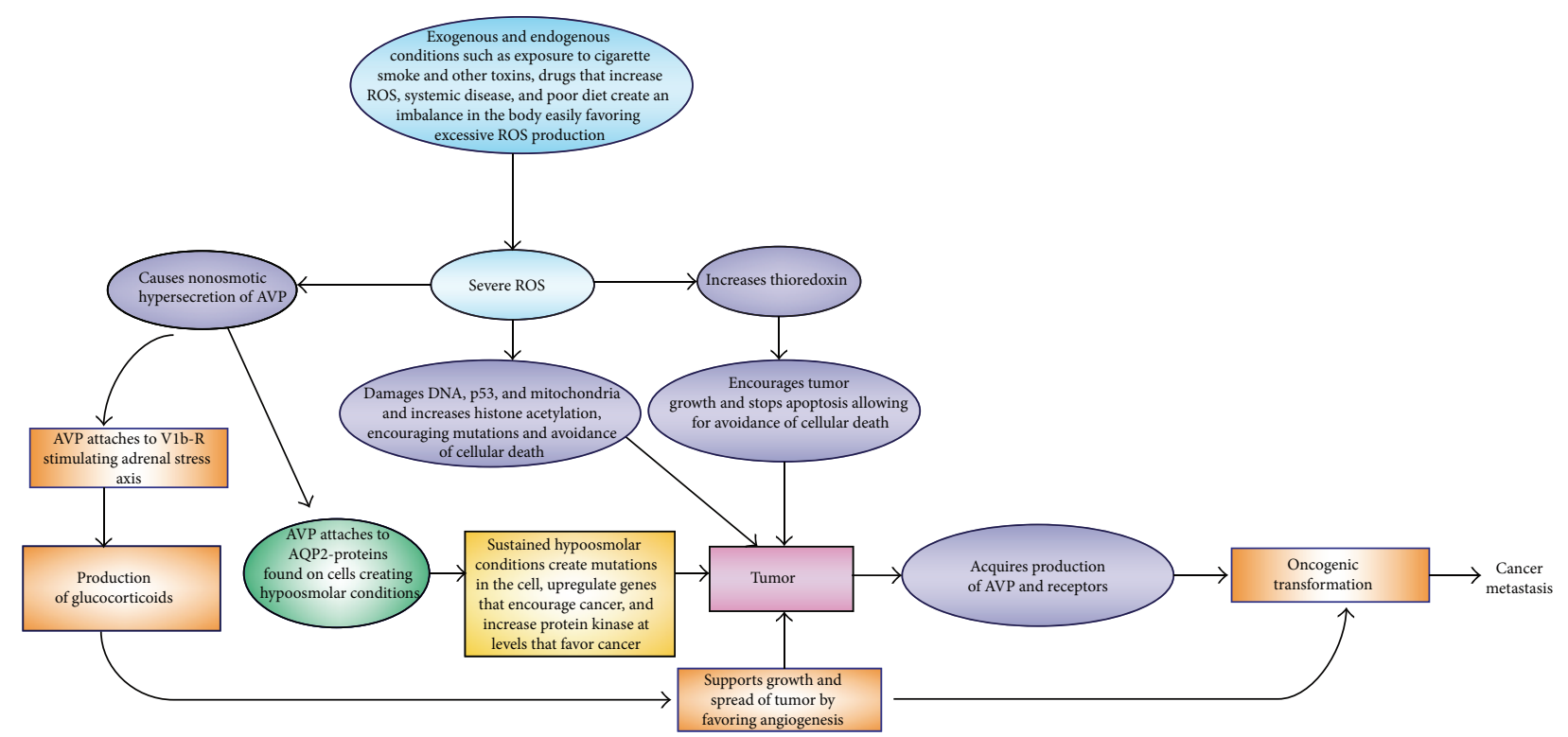

FIGURE 1: It appears that the "altered water system" is capable of maintaining a balance between dysregulated proliferation, cell cycle arrest, apoptosis, and genomic instability allowing both the transformation of a cell into a cancerous cell and cancer metastasis.

\section{Conclusion}

Exploration of this "altered water system" requires a large initial investment in future research; however, the knowledge obtained from this research should significantly enhance the prevention and treatment of cancers. General suggestions for future research include analyses of the influence of water and osmolarity on gene expression and the potential oncogenic effects of the synergism of ROS (and other signaling molecules) with the overproduction of AVP.

\section{Conflict of Interests}

The authors declare that there is no conflict of interests regarding the publication of this paper.

\section{References}

[1] Y. Levy and J. N. Onuchic, "Water and proteins: a love-hate relationship," Proceedings of the National Academy of Sciences of the United States of America, vol. 101, no. 10, pp. 3325-3326, 2004.

[2] Z. Szolnoki, "A dynamically changing intracellular water network serves as a universal regulator of the cell: the watergoverned cycle," Biochemical and Biophysical Research Communications, vol. 357, no. 2, pp. 331-334, 2007.

[3] M. M. Chaves, J. Flexas, and C. Pinheiro, "Photosynthesis under drought and salt stress: regulation mechanisms from whole plant to cell," Annals of Botany, vol. 103, no. 4, pp. 551-560, 2009.

[4] S. Ma, Q. Gong, and H. J. Bohnert, "Dissecting salt stress pathways," Journal of Experimental Botany, vol. 57, no. 5, pp. 1097-1107, 2006.

[5] K. Shinozaki and K. Yamaguchi-Shinozaki, "Gene networks involved in drought stress response and tolerance," The Journal of Experimental Botany, vol. 58, no. 2, pp. 221-227, 2007.
[6] N. Mutsuga, T. Shahar, J. G. Verbalis, C. C. Xiang, M. J. Brownstein, and H. Gainer, "Regulation of gene expression in magnocellular neurons in rat supraoptic nucleus during sustained hypoosmolality," Endocrinology, vol. 146, no. 3, pp. 12541267, 2005.

[7] S. J. Somponpun and C. D. Sladek, "Osmotic regulation of estrogen receptor- $\beta$ in rat vasopressin and oxytocin neurons," The Journal of Neuroscience, vol. 23, no. 10, pp. 4261-4269, 2003.

[8] S. H. Sleigh, P. R. Seavers, A. J. Wilkinson, J. E. Ladbury, and J. R. H. Tame, "Crystallographic and calorimetric analysis of peptide binding to OppA protein," Journal of Molecular Biology, vol. 291, no. 2, pp. 393-415, 1999.

[9] S. Bass, V. Sorrells, and P. Youderian, "Mutant Trp repressors with new DNA-binding specificities," Science, vol. 241, no. 4876, pp. 240-245, 1988.

[10] A. Joachimiak, T. E. Haran, and P. B. Sigler, "Mutagenesis supports water mediated recognition in the trp repressoroperator system," The EMBO Journal, vol. 13, no. 2, pp. 367-372, 1994.

[11] J. W. Schwabe, "The role of water in protein-DNA interactions," Current Opinion in Structural Biology, vol. 7, pp. 126-134, 1997.

[12] R. W. Schevitz, Z. Otwinowski, A. Joachimiak, C. L. Lawson, and P. B. Sigler, "The three-dimensional structure of trp repressor," Nature, vol. 317, no. 6040, pp. 782-786, 1985.

[13] Z. Shakked, G. Guzikevich-Guerstein, F. Frolow, D. Rabinovich, A. Joachimiak, and P. B. Sigler, "Determinants of repressor/operator recognition from the structure of the trp operator binding site," Nature, vol. 368, no. 6470, pp. 469-473, 1994.

[14] P. B. Sigler, "The surprising chemistry of specific protein nucleic acid interfaces," in Proceedings of the Robert A Welch Foundation 37th Conference on Chemical Research, Houston, Tex, USA, October 1993.

[15] R.-G. Zhang, A. Joachimiak, C. L. Lawson, R. W. Schevitz, Z. Otwinowski, and P. B. Sigler, "The crystal structure of trp aporepressor at $1.8 \AA$ shows how binding tryptophan enhances DNA affinity," Nature, vol. 327, no. 6123, pp. 591-597, 1987. 
[16] O. Filhol and C. Cochet, "Protein kinase CK2 and cancer: further clues are accumulating," Bulletin du Cancer, vol. 89, no. 3, pp. 261-265, 2002.

[17] K. Niefind, M. Putter, B. Guerra, O.-G. Issinger, and D. Schomburg, "GTP plus water mimic ATP in the active site of protein kinase CK2," Nature Structural Biology, vol. 6, no. 12, pp. 1100$1103,1999$.

[18] L. L. Wong and J. G. Verbalis, "Systemic diseases associated with disorders of water homeostasis," Endocrinology and Metabolism Clinics of North America, vol. 31, no. 1, pp. 121-140, 2002.

[19] J. Laycock, "Introduction to vasopressin," in Perspectives on Vasopressin, J. Laycock, Ed., pp. 1-20, Imperial College Press, London, UK, 2010.

[20] J. G. Verbalis, "Disorders of body water homeostasis," Best Practice and Research: Clinical Endocrinology and Metabolism, vol. 17, no. 4, pp. 471-503, 2003.

[21] R. St-Louis, C. Parmentier, D. Raison, V. Grange-Messent, and H. Hardin-Pouzet, "Reactive oxygen species are required for the hypothalamic osmoregulatory response," Endocrinology, vol. 153, no. 3, pp. 1317-1329, 2012.

[22] R. St-Louis, C. Parmentier, V. Grange-Messent, S. MhaoutyKodja, and H. Hardin-Pouzet, "Reactive oxygen species are physiological mediators of the noradrenergic signaling pathway in the mouse supraoptic nucleus," Free Radical Biology and Medicine, vol. 71, pp. 231-239, 2014.

[23] O. F. Pomerleau, "Nicotine and the central nervous system: biobehavioral effects of cigarette smoking," The American Journal of Medicine, vol. 93, no. 1, pp. 2S-7S, 1992.

[24] P. Chiodera, L. Capretti, M. Marchesi et al., "Abnormal arginine vasopressin response to cigarette smoking and metoclopramide (but not to insulin-induced hypoglycemia) in elderly subjects," Journals of Gerontology, vol. 46, no. 1, pp. M6-M10, 1991.

[25] L. E. Seyler Jr., O. F. Pomerleau, J. B. Fertig, D. Hunt, and K. Parker, "Pituitary hormone response to cigarette smoking," Pharmacology, Biochemistry and Behavior, vol. 24, no. 1, pp. 159$162,1986$.

[26] X.-M. Ma, A. Levy, and S. L. Lightman, "Emergence of an isolated arginine vasopressin (AVP) response to stress after repeated restraint: a study of both AVP and corticotropinreleasing hormone messenger ribonucleic acid (RNA) and heteronuclear RNA," Endocrinology, vol. 138, no. 10, pp. 43514357, 1997.

[27] H. Taivainen, K. Laitinen, R. Tähtelä, K. Kiianmaa, and M. J. Välimäki, "Role of plasma vasopressin in changes of water balance accompanying acute alcohol intoxication," Alcoholism: Clinical and Experimental Research, vol. 19, no. 3, pp. 759-762, 1995.

[28] G. Bedogni, A. Borghi, and N. Battistini, "Body water distribution and disease," Acta Diabetologica, vol. 40, supplement 1, pp. S200-S202, 2003.

[29] G. Miltiadous, D. Christidis, M. Kalogirou, and M. Elisaf, "Causes and mechanisms of acid-base and electrolyte abnormalities in cancer patients," European Journal of Internal Medicine, vol. 19, no. 1, pp. 1-7, 2008.

[30] E. Y. M. Sum, B. Peng, X. Yu et al., "The LIM domain protein LMO4 interacts with the cofactor CtIP and the tumor suppressor BRCA1 and inhibits BRCA1 activity," The Journal of Biological Chemistry, vol. 277, no. 10, pp. 7849-7856, 2002.

[31] W. G. North, S. Pai, A. Friedmann, X. Yu, M. Fay, and V. Memoli, "Vasopressin gene related products are markers of human breast cancer," Breast Cancer Research and Treatment, vol. 34, pp. 229235, 1995.
[32] W. G. North, S. Pai, A. Friedmann, X. Yu, M. Fay, and V. Memoli, "Erratum in: Vasopressin gene related products are markers of human breast cancer," Breast Cancer Research and Treatment, vol. 36, no. 1, p. 99, 1995.

[33] National Cancer Institute, Understanding cancer series: estrogen receptors/SERMs, http://www.cancer.gov/.

[34] D. Bolignano, M. A. Medici, G. Coppolino et al., "Aquaretic inhibits renal cancer proliferation: role of vasopressin receptor2 (V2-R)," Urologic Oncolog, vol. 28, no. 6, pp. 642-647, 2010.

[35] W. G. North, "Gene regulation of vasopressin and vasopressin receptors in cancer," Experimental Physiology, vol. 85, pp. 27S40S, 2000.

[36] J. Rutishauser and P. Kopp, "Aquaporin-2 water channel mutations and nephrogenic diabetes insipidus: new variations on a theme," European Journal of Endocrinology, vol. 140, no. 2, pp. 137-139, 1999.

[37] B. P. Keegan, B. L. Akerman, C. Péqueux, and W. G. North, "Provasopressin expression by breast cancer cells: implications for growth and novel treatment strategies," Breast Cancer Research and Treatment, vol. 95, no. 3, pp. 265-277, 2006.

[38] J. Laycock, "Understanding the role of vasopressin in the hypothalamo-pituitary adrenal axis," in Perpsectives on Vasopressin, J. Buckingman, Ed., pp. 230-256, Imperial College Press, London, UK, 2010.

[39] D. Franchimont, "Overview of the actions of glucocorticoids on the immune response: a good model to characterize new pathways of immunosuppression for new treatment strategies," Annals of the New York Academy of Sciences, vol. 1024, pp. 124$137,2004$.

[40] K. E. de Visser, A. Eichten, and L. M. Coussens, "Paradoxical roles of the immune system during cancer development," Nature Reviews Cancer, vol. 6, no. 1, pp. 24-37, 2006.

[41] S. J. Lolait, L. Q. Stewart, D. S. Jessop, W. S. Young III, and A. M. O'Carroll, "The hypothalamic-pituitary-adrenal axis response to stress in mice lacking functional vasopressin V1b receptors," Endocrinology, vol. 148, no. 2, pp. 849-856, 2007.

[42] Z. Sapolsky, "Immunity, stress, and disease," in Why Zebras Don't Get Ulcers? p. 539, Henry Holt, New York, NY, USA, 3rd edition, 2004.

[43] G. Alonso, E. Gallibert, C. Lafont, and G. Guillon, "Intrahypothalamic angiogenesis induced by osmotic stimuli correlates with local hypoxia: a potential role of confined vasoconstriction induced by dendritic secretion of vasopressin," Endocrinology, vol. 149, no. 9, pp. 4279-4288, 2008.

[44] C. K. Sen and L. Packer, "Thiol homeostasis and supplements in physical exercise," American Journal of Clinical Nutrition, vol. 72, no. 2, pp. 653S-669S, 2000.

[45] A. E. Aust and J. F. Eveleigh, "Mechanisms of DNA oxidation," Proceedings of the Society for Experimental Biology and Medicine, vol. 222, no. 3, pp. 246-252, 1999.

[46] B. Halliwell, "Antioxidants in human health and disease," Annual Review of Nutrition, vol. 16, pp. 33-50, 1996.

[47] B. Halliwell, J. M. C. Gutteridge, and C. E. Cross, "Free radicals, antioxidants, and human disease: where are we now?" The Journal of Laboratory and Clinical Medicine, vol. 119, no. 6, pp. 598-620, 1992.

[48] M. Golden, "Free radicals and the aetiology of Kwashiorkor," Biochemist, 1994.

[49] E. Alirol and J. C. Martinou, "Mitochondria and cancer: is there a morphological connection?" Oncogene, vol. 25, no. 34, pp. 4706-4716, 2006. 
[50] S. Y. Archer and R. A. Hodin, "Histone acetylation and cancer," Current Opinion in Genetics and Development, vol. 9, no. 2, pp. 171-174, 1999.

[51] D. Patel and D. Allis, Linking Histones and Cancer: DNA's Packaging Proteins Hold Clues to How Our Genes Work and What Causes Them to Malfunction, Memorial Sloan-Kettering Cancer Center, 2010, http://www.mskcc.org/mskcc/html/95063.cfm.

[52] P. T. Schumacker, "Reactive oxygen species in cancer cells: live by the sword, die by the sword," Cancer Cell, vol. 10, no. 3, pp. 175-176, 2006.

[53] W. Davis Jr., Z. Ronai, and K. D. Tew, "Cellular thiols and reactive oxygen species in drug-induced apoptosis," Journal of Pharmacology and Experimental Therapeutics, vol. 296, no. 1, pp. 1-6, 2001.

[54] G. Powis, D. Mustacich, and A. Coon, "The role of the redox protein thioredoxin in cell growth and cancer," Free Radical Biology and Medicine, vol. 29, no. 3-4, pp. 312-322, 2000. 

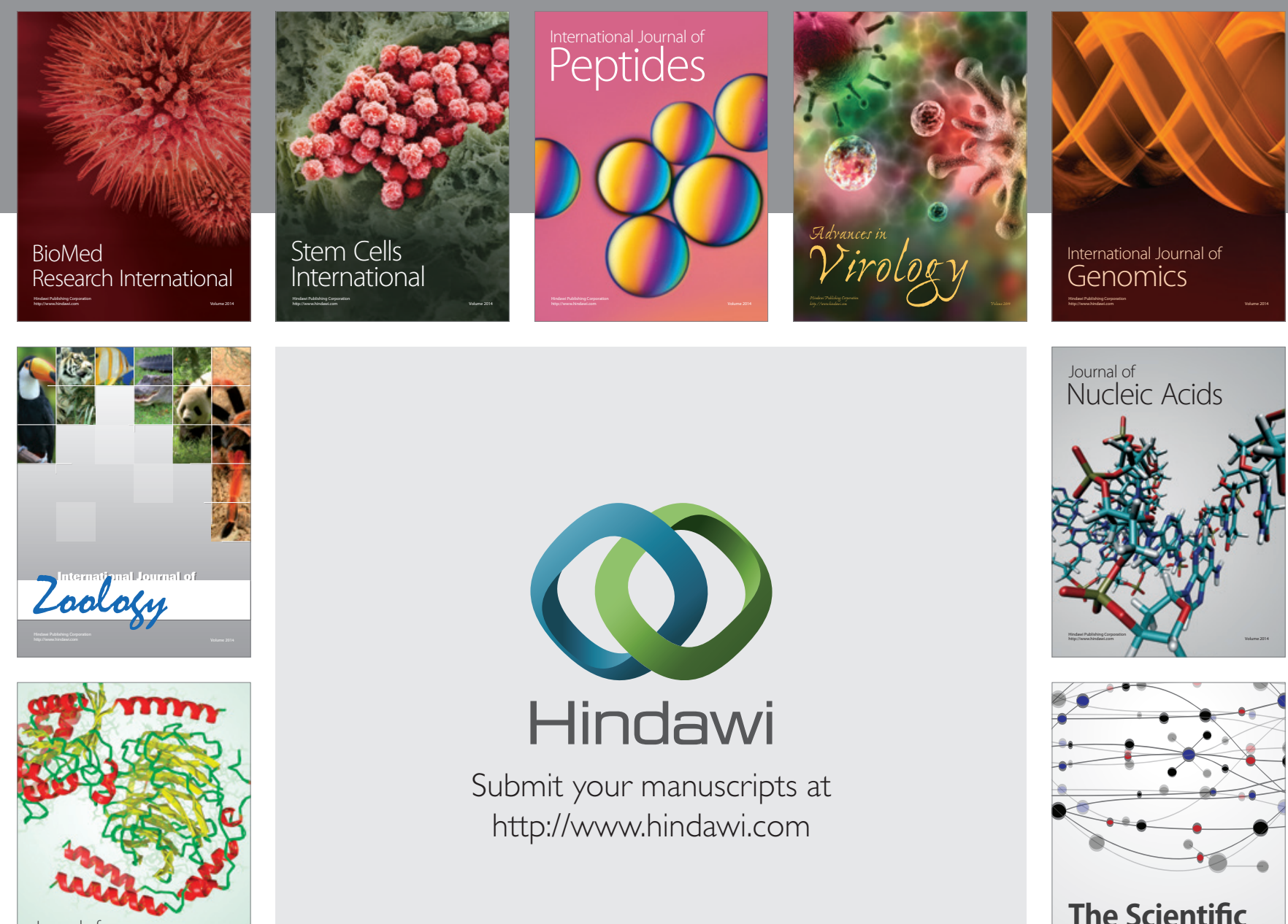

Submit your manuscripts at

http://www.hindawi.com

Journal of
Signal Transduction
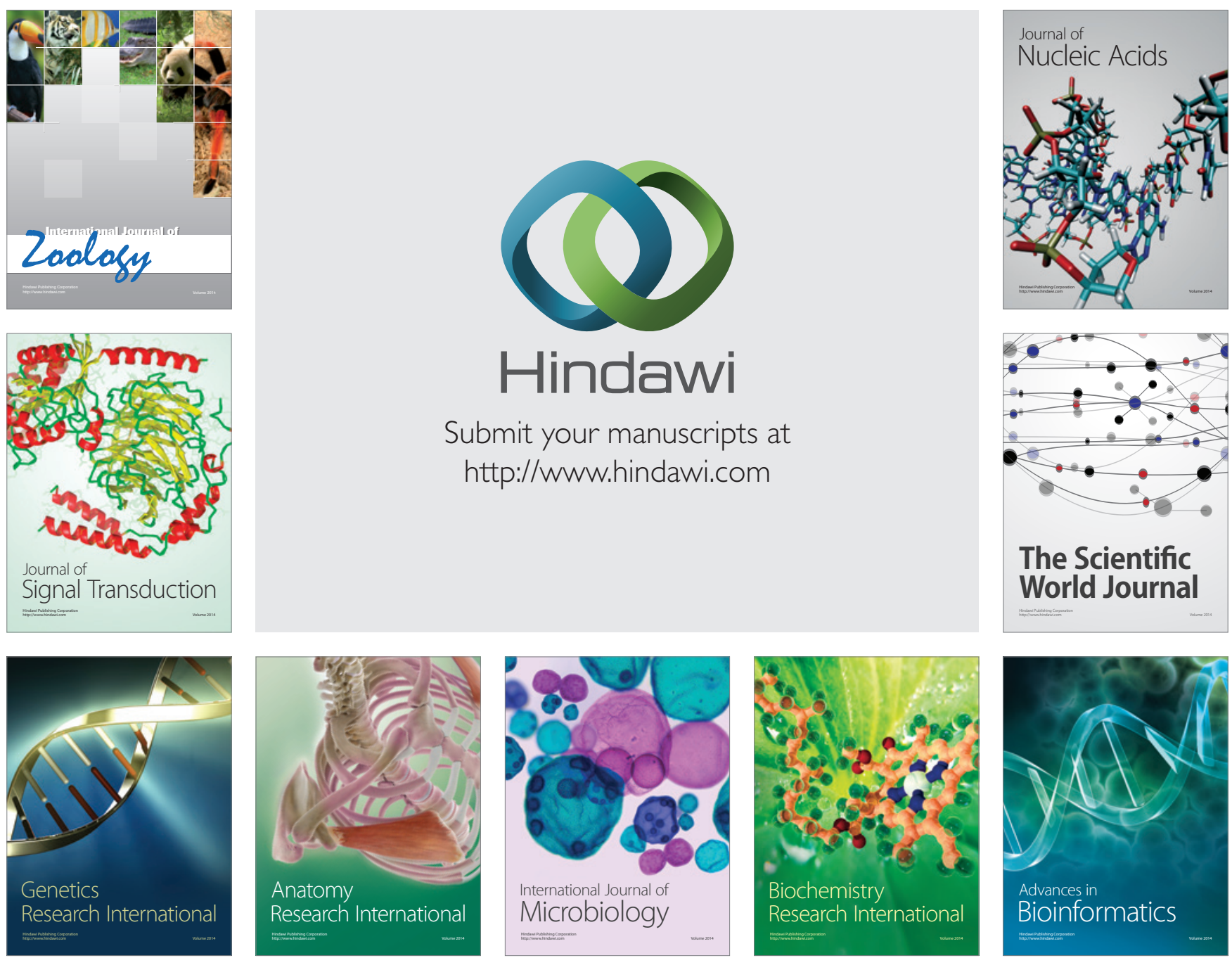

The Scientific World Journal
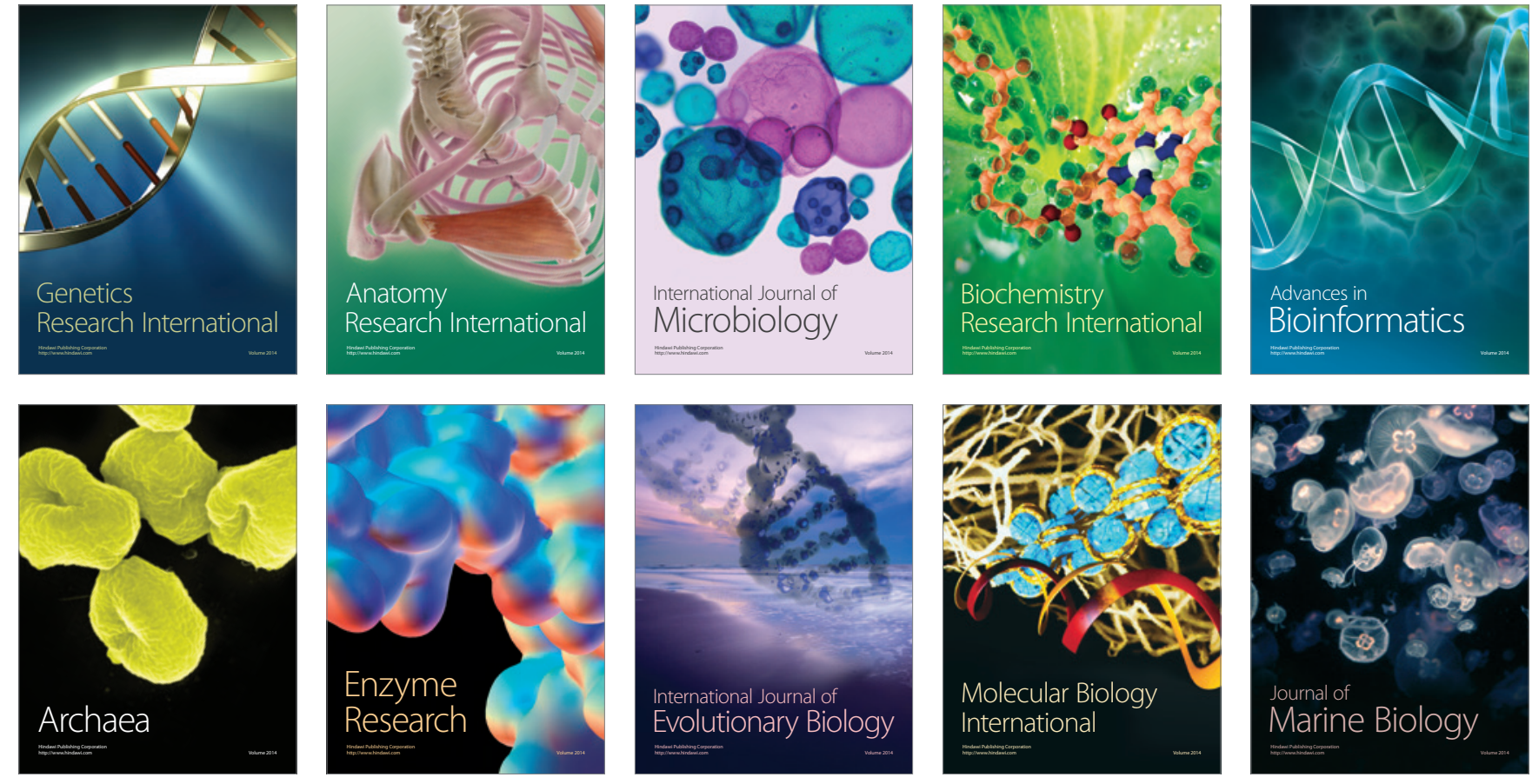